ULB-TH 99/01

April 1999

\title{
Phenomenological evidence for the energy dependence of the $\eta-\eta /$ mixing angle
}

\author{
R. Escribano" and J.-M. Frèref \\ Service de Physique Théorique, Université Libre de Bruxelles, CP 225, B-1050 Bruxelles, \\ Belgium
}

\begin{abstract}
A phenomenological analysis on various decay processes is performed using an energy-dependent $\eta-\eta /$ mixing angle scheme. Special attention is given to the electromagnetic couplings between lowest-lying vector and pseudoscalar mesons. The agreement between our predictions and the experimental values is remarkable. This analysis opens a connection to two-angle fits in the $\eta-\eta /$ sector.
\end{abstract}

\footnotetext{
${ }^{1}$ Chercheur IISN.

${ }^{2}$ Directeur de recherches du FNRS.
} 


\section{Introduction}

The $\eta-\eta \prime$ mixing angle has become one of the most interesting $S U(3)$-breaking hadronic parameters to measure since $S U(3)$ symmetry was proposed [1]. Several exhaustive analyses surveying many different processes have been performed along the years [2, 3, 4, 5]. The values presented for the mixing angle range from $-20^{\circ}$ to $-10^{\circ}$. In all those previous analyses the dependence with energy of the $\eta-\eta$ ' mixing angle has been neglected. The main reason for such simplification is that there is not yet an established theoretical framework where the $\eta^{\prime}$ could be well incorporated. Recently, a combination of Chiral Perturbation Theory $(\chi \mathrm{PT})$ together with a simultaneous expansion in $1 / N_{c}$ (in order to take into account the axial $U_{A}(1)$ anomaly) has been proposed for describing the $\eta \prime$ dynamics [6, 7]. In this so-called Extended- $\chi \mathrm{PT}$ $(\mathrm{E} \chi \mathrm{PT})$, the energy dependence of the $\eta-\eta \prime$ mixing angle could be traced. However, the $q^{2}$-dependence expected from loop corrections starts at orders that cannot be actually computed due to the proliferation of unknown parameters [8]. In the analogous case of $\pi^{0}-\eta$ mixing, $\chi \mathrm{PT}$ alone provides a valuable framework where the $q^{2}$-dependence can be followed, showing that the $\pi^{0}-\eta$ mixing angle is modified by an $8 \%$ from $q^{2}=m_{\pi}^{2}$ to $q^{2}=m_{\eta}^{2}$ [9]. In principle, there is no reason for avoiding a similar behaviour for the $\eta-\eta \prime$ case.

Yet in Ref. [四, an agreement with experimental data, including $J / \psi$ radiative decays, was achieved using an energy-independent mixing angle, in contrast with $\chi \mathrm{PT}$ where the energy-independent parametrization fails in describing those radiative decays. This difference is due to the fact that the approach of Refs. [4, 10] contains ab initio the axial anomaly. Similarly, the $\mathrm{E} \chi \mathrm{PT}$ includes the effect of the axial anomaly through a perturbative expansion, allowing the presence of the pseudoscalar singlet as an additional degree of freedom, and thus the possibility of considering an energy dependence for the $\eta$ - $\eta$ ' mixing angle.

In this letter we extend a previous analysis of various decay processes assuming an energy dependence of the $\eta-\eta$ ' mixing angle and check how this modification improves the results of Ref. [4. We do not use any theoretical prejudice about the explicit $q^{2}$-dependence. We simply assume that the value

of the mixing angle at $q^{2}=m_{\eta}^{2}$ can differ from the value at $q^{2}=m_{\eta^{\prime}}^{2}$. In Section 2, we shortly introduce the notation used in the analysis. In Sec. 3, we compute the radiative decays $(\eta, \eta \prime) \rightarrow \gamma \gamma$ and the ratio $R_{J / \psi} \equiv \Gamma(J / \psi \rightarrow$ 
$\eta \prime \gamma / \eta \gamma)$ in the energy-dependent scheme in order to obtain the preferred values for the mixing angles involved in the analysis. Sec. 4 is devoted to the consequences of our approach in the context of the radiative decays of lowestlying vector and pseudoscalar mesons, $V \rightarrow P \gamma$ and $P \rightarrow V \gamma$, respectively. Finally, in Sec. 5, we present our conclusions.

\section{Notation}

The $\eta-\eta /$ mixing angle in the octet-singlet basis allowing for an energy dependence is written as

$$
\begin{gathered}
|\eta\rangle=c \theta_{\eta}\left|\eta_{8}\right\rangle-s \theta_{\eta}\left|\eta_{0}\right\rangle, \\
\left|\eta^{\prime}\right\rangle=s \theta_{\eta^{\prime}}\left|\eta_{8}\right\rangle+c \theta_{\eta^{\prime}}\left|\eta_{0}\right\rangle,
\end{gathered}
$$

where $\theta_{\eta}$ and $\theta_{\eta^{\prime}}$ are defined as the values of the mixing angle $\theta\left(q^{2}\right)$ at $q^{2}=m_{\eta}^{2}$ and $q^{2}=m_{\eta^{\prime}}^{2}$ respectively. Due to the assumed energy dependence, the orthogonality of the physical states in Eq. (1) is no longer satisfied, contrary to the usual energy-independent scheme where $\theta_{\eta}=\theta_{\eta^{\prime}} \equiv \theta$ is assumed [1].

The pseudoscalar decay constants $f_{P}^{i}(i=8,0 ; P=\eta, \eta \prime)$ are defined as

$$
\left\langle 0\left|A_{\mu}^{i}\right| P(p)\right\rangle=i f_{P}^{i} p_{\mu}
$$

where $A_{\mu}^{8,0}$ are the octet and singlet axial-vector currents whose divergences are

$$
\begin{gathered}
\partial^{\mu} A_{\mu}^{8}=\frac{2}{\sqrt{6}}\left(m_{u} \bar{u} i \gamma_{5} u+m_{d} \bar{d} i \gamma_{5} d-2 m_{s} \bar{s} i \gamma_{5} s\right), \\
\partial^{\mu} A_{\mu}^{0}=\frac{2}{\sqrt{3}}\left(m_{u} \bar{u} i \gamma_{5} u+m_{d} \bar{d} i \gamma_{5} d+m_{s} \bar{s} i \gamma_{5} s\right)+\frac{1}{\sqrt{3}} \frac{3 \alpha_{s}}{4 \pi} G_{\mu \nu}^{a} \tilde{G}^{a, \mu \nu},
\end{gathered}
$$

where $G_{\mu \nu}^{a}$ is the gluonic field-strength tensor and $\tilde{G}_{\mu \nu}^{a} \equiv \frac{1}{2} \epsilon_{\mu \nu \alpha \beta} G^{a, \alpha \beta}$ its dual. The divergence of the matrix elements (2) are then written as

$$
\left\langle 0\left|\partial^{\mu} A_{\mu}^{i}\right| P\right\rangle=f_{P}^{i} m_{P}^{2},
$$

where $m_{P}$ is the mass of the pseudoscalar meson. 
In our analysis, we assume that the pseudoscalar decay constants will follow the same mixing pattern as the particle state mixing does ${ }^{3}$ (see Eq. (1)):

$$
\left(\begin{array}{cc}
f_{\eta}^{8} & f_{\eta}^{0} \\
f_{\eta^{\prime}}^{8} & f_{\eta^{\prime}}^{0}
\end{array}\right)=\left(\begin{array}{cc}
f_{8} c \theta_{\eta} & -f_{0} s \theta_{\eta} \\
f_{8} s \theta_{\eta^{\prime}} & f_{0} c \theta_{\eta^{\prime}}
\end{array}\right) .
$$

Neglecting the contribution of the up and down quark masses, as in Ref. [10], the matrix elements of the chiral anomaly between the vacuum and $(\eta, \eta \prime)$ states are

$$
\begin{aligned}
\left\langle 0\left|\frac{3 \alpha_{s}}{4 \pi} G \tilde{G}\right| \eta\right\rangle & =\sqrt{\frac{3}{2}} m_{\eta}^{2}\left(f_{8} c \theta_{\eta}-\sqrt{2} f_{0} s \theta_{\eta}\right), \\
\left\langle 0\left|\frac{3 \alpha_{s}}{4 \pi} G \tilde{G}\right| \eta \prime\right\rangle & =\sqrt{\frac{3}{2}} m_{\eta^{\prime}}^{2}\left(f_{8} s \theta_{\eta^{\prime}}+\sqrt{2} f_{0} c \theta_{\eta^{\prime}}\right) .
\end{aligned}
$$

\section{Experimental values for the $\theta_{\eta}$ and $\theta_{\eta^{\prime}}$ mix- ing angles}

In order to reach some predictions from our energy-dependent mixing angle analysis we must first know the values of $\theta_{\eta}$ and $\theta_{\eta^{\prime}}$ preferred by the experimental data. We will use as constraints the experimental decay widths of $(\eta, \eta \prime) \rightarrow \gamma \gamma$ [迺]

$$
\begin{aligned}
\Gamma(\eta \rightarrow \gamma \gamma) & =(0.46 \pm 0.04) \mathrm{keV} \\
\Gamma(\eta \prime \rightarrow \gamma \gamma) & =(4.27 \pm 0.19) \mathrm{keV}
\end{aligned}
$$

Generalizing the PCAC result for the $\pi^{0} \rightarrow \gamma \gamma$ decay, one assumes that the interpolating fields $\eta$ and $\eta$ ' can be related via PCAC with the axial-vector currents (see e.g. Refs. [12, 13]) in the following way

$$
\begin{aligned}
& \eta(x)=\frac{1}{m_{\eta}^{2}} \frac{f_{\eta^{\prime}}^{0} \partial^{\mu} A_{\mu}^{8}(x)-f_{\eta^{\prime}}^{8} \partial^{\mu} A_{\mu}^{0}(x)}{f_{\eta^{\prime}}^{0} f_{\eta}^{8}-f_{\eta^{\prime}}^{8} f_{\eta}^{0}}, \\
& \eta \prime(x)=\frac{1}{m_{\eta^{\prime}}^{2}} \frac{f_{\eta}^{0} \partial^{\mu} A_{\mu}^{8}(x)-f_{\eta}^{8} \partial^{\mu} A_{\mu}^{0}(x)}{f_{\eta}^{0} f_{\eta^{\prime}}^{8}-f_{\eta}^{8} f_{\eta^{\prime}}^{0}} .
\end{aligned}
$$

\footnotetext{
${ }^{3}$ In Eq. (5), we assume for definiteness that only the mixing angle $\theta$ (and not the decay constants $f_{8,0}$ ) is energy-dependent. In fact, the fit to the experimental data only deals with the energy dependence of the products $f_{P}^{i}$, and not with the energy dependence of $\theta$ and $f_{8,0}$ separately. A more refined analysis using for instance off-shell processes might help to distinguish between both energy dependences.

${ }^{4}$ We choose such constrains because those decays are well understood in terms of the electromagnetic anomaly (see e.g. Ref. [11]).
} 
This leads tof

$$
\begin{aligned}
& \Gamma(\eta \rightarrow \gamma \gamma)=\frac{\alpha^{2} m_{\eta}^{3}}{96 \pi^{3}}\left(\frac{f_{\eta^{\prime}}^{0}-2 \sqrt{2} f_{\eta^{\prime}}^{8}}{f_{\eta^{\prime}}^{0} f_{\eta}^{8}-f_{\eta^{\prime}}^{8} f_{\eta}^{0}}\right)^{2}=\frac{\alpha^{2} m_{\eta}^{3}}{96 \pi^{3}}\left(\frac{c \theta_{\eta^{\prime}} / f_{8}-2 \sqrt{2} s \theta_{\eta^{\prime}} / f_{0}}{c \theta_{\eta^{\prime}} c \theta_{\eta}+s \theta_{\eta^{\prime}} s \theta_{\eta}}\right)^{2}, \\
& \Gamma\left(\eta^{\prime} \rightarrow \gamma \gamma\right)=\frac{\alpha^{2} m_{\eta^{\prime}}^{3}}{96 \pi^{3}}\left(\frac{f_{\eta}^{0}-2 \sqrt{2} f_{\eta}^{8}}{f_{\eta}^{0} f_{\eta^{\prime}}^{8}-f_{\eta}^{8} f_{\eta^{\prime}}^{0}}\right)^{2}=\frac{\alpha^{2} m_{\eta^{\prime}}^{3}}{96 \pi^{3}}\left(\frac{s \theta_{\eta} / f_{8}+2 \sqrt{2} c \theta_{\eta} / f_{0}}{c \theta_{\eta^{\prime}} c \theta_{\eta}+s \theta_{\eta^{\prime}} s \theta_{\eta}}\right)^{2} .
\end{aligned}
$$

Because of the four unknown parameters $\left(\theta_{\eta}, \theta_{\eta^{\prime}}, f_{8}\right.$ and $\left.f_{0}\right)$ that appear in Eq. (9), in order to get their allowed values we need two additional constraints (apart from the experimental constraints in Eq. (7)). On the one hand, we will use the well established prediction of $\chi \mathrm{PT}: f_{8}=1.28 f_{\pi}\left(f_{\pi}=132 \mathrm{MeV}\right)$ as a theoretical constrain. On the other hand, we will use the experimental value of the ratio [1]

$$
R_{J / \psi} \equiv \frac{\Gamma(J / \psi \rightarrow \eta \prime \gamma)}{\Gamma(J / \psi \rightarrow \eta \gamma)}=5.0 \pm 0.6
$$

According to Ref. [14, the radiative $J / \psi \rightarrow P \gamma$ decays are dominated by

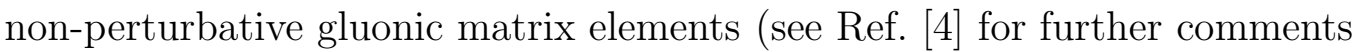
on the accuracy of this statement):

$$
R_{J / \psi}=\left|\frac{\langle 0|G \tilde{G}| \eta \prime\rangle}{\langle 0|G \tilde{G}| \eta\rangle}\right|^{2}\left(\frac{p_{\eta^{\prime}}}{p_{\eta}}\right)^{3}
$$

where $p_{P}=M_{J / \psi}\left(1-m_{P}^{2} / M_{J / \psi}^{2}\right) / 2$ is the three-momentum of the $P$-meson in the rest frame of the decaying $J / \psi$ (with mass $M_{J / \psi}$ ). Using Eq. (6) one gets

$$
R_{J / \psi}=\left|\frac{m_{\eta^{\prime}}^{2}\left(f_{\eta^{\prime}}^{8}+\sqrt{2} f_{\eta^{\prime}}^{0}\right)}{m_{\eta}^{2}\left(f_{\eta}^{8}+\sqrt{2} f_{\eta}^{0}\right)}\right|^{2}\left(\frac{p_{\eta^{\prime}}}{p_{\eta}}\right)^{3}=\left(\frac{m_{\eta^{\prime}}^{2}\left(f_{8} s \theta_{\eta^{\prime}}+\sqrt{2} f_{0} c \theta_{\eta^{\prime}}\right)}{m_{\eta}^{2}\left(f_{8} c \theta_{\eta}-\sqrt{2} f_{0} s \theta_{\eta}\right)}\right)^{2}\left(\frac{p_{\eta^{\prime}}}{p_{\eta}}\right)^{3}
$$

Comparing the experimental values of $\Gamma(\eta, \eta \prime \rightarrow \gamma \gamma)$ and $R_{J / \psi}$ with the theoretical predictions shown in Eqs. (9) and (12), one obtains

$$
\begin{gathered}
\theta_{\eta}=(-6.5 \pm 2.5)^{\circ}, \\
\theta_{\eta^{\prime}}=(-23.1 \pm 3.0)^{\circ}, \\
f_{0}=(1.31 \pm 0.07) f_{\pi} .
\end{gathered}
$$

\footnotetext{
${ }^{5}$ Note that if one assumes an energy-independent parameterization of the mixing angle $\left(\theta_{\eta}=\theta_{\eta^{\prime}} \equiv \theta\right)$ the standard result is obtained (see e.g. Ref. [1]).
} 
The previous values constitute the first result of the present analysis. It is worth noting that the $\theta_{\eta}$ and $\theta_{\eta^{\prime}}$ mixing angle values are different at the $3 \sigma$ level, while the value of the pseudoscalar decay constant $f_{0}$ remains compatible with other results. Eq. (13) shows that the difference between the value of the mixing angle at $q^{2}=m_{\eta}^{2}$ and $q^{2}=m_{\eta^{\prime}}^{2}$ is of the order of $250 \%$, a huge difference compared to the $8 \%$ corresponding to the $\pi^{0}-\eta q^{2}$-dependence. We are not able to argue the reason for such a different behaviour because the values presented in Eq. (13) are the result of a phenomenological analysis. Nevertheless, we must make some comments that could be relevant for understanding the origin of that difference: on the one hand, we assume that all the energy dependence is included in the mixing angle (see Sec. 2). On the other hand, the $\eta-\eta$ ' sector is different from the $\pi^{0}-\eta$ sector because of the major rôle played by the axial anomaly in the singlet mass (from which the mixing angle is defined), that prevents the singlet from being a Goldstone boson in the chiral limit. This fact induces a different behaviour on the $\eta-\eta \prime$ mixing angle, and presumably on its energy dependence, with respect to its $\pi^{0}-\eta$ counterpart. However, the definite resolution of this dilemma would come from the analytical calculation of the energy dependence of the mixing angle in the framework of the $\mathrm{E} \chi \mathrm{PT}$, that would provide a valuable comparison with phenomenological analyses, comparison that is already available for the $\pi^{0}-\eta$ case.

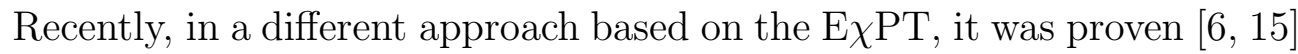
that at next-to-leading order the pseudoscalar decay constants $f_{P}^{i}$ could not be explained in terms of just one mixing angle, but two different mixing angles would be needed, one associated to the octet and the other to the singlet:

$$
\left(\begin{array}{cc}
f_{\eta}^{8} & f_{\eta}^{0} \\
f_{\eta^{\prime}}^{8} & f_{\eta^{\prime}}^{0}
\end{array}\right)=\left(\begin{array}{cc}
\tilde{f}_{8} c \theta_{8} & -\tilde{f}_{0} s \theta_{0} \\
\tilde{f}_{8} s \theta_{8} & \tilde{f}_{0} c \theta_{0}
\end{array}\right) .
$$

In that theoretical framework one gets $\left(\tilde{f}_{8}=1.28 f_{\pi}\right.$ fixed from $\left.\chi \mathrm{PT}\right)$

$$
\theta_{8}=-20.5^{\circ}, \quad \theta_{0} \simeq-4^{\circ}, \quad \tilde{f}_{0} \simeq 1.25 f_{\pi} .
$$

Ref. [16] uses the hypothesis that the four wave functions associated to the pseudoscalar mesons, from which the pseudoscalar decay constants are de-

\footnotetext{
${ }^{6}$ The original notation in Refs. 6, 15] for the octet and singlet decay constants $f_{8}$ and $f_{0}$ is slightly modified here to $\tilde{f}_{8}$ and $f_{0}$ in order to avoid a confusion between the two schemes (see Eqs. (5) and (14)).
} 
fined, are different. Then, two different mixing angles for the decay constants must be introduced. Their analysis gives

$$
\theta_{8}=-22.2^{\circ}, \quad \theta_{0} \simeq-9.1^{\circ}, \quad \tilde{f}_{0} \simeq 1.20 f_{\pi} .
$$

It is straightforward to establish the link between our results in Eq. (13) and the octet-singlet two-angle mixing scheme for the pseudoscalar decay constants

$$
\begin{gathered}
\tan \theta_{8}=\frac{\sin \theta_{\eta^{\prime}}}{\cos \theta_{\eta}}, \quad \tan \theta_{0}=\frac{\sin \theta_{\eta}}{\cos \theta_{\eta^{\prime}}}, \\
\tilde{f}_{0}=f_{0} \sqrt{s^{2} \theta_{\eta}+c^{2} \theta_{\eta^{\prime}}},
\end{gathered}
$$

yielding

$$
\theta_{8}=(-21.5 \pm 2.4)^{\circ}, \theta_{0}=(-7.0 \pm 2.7)^{\circ}, \tilde{f}_{0}=(1.21 \pm 0.07) f_{\pi} .
$$

These values are fully compatible with those obtained in Refs. [6, 16]. The pseudoscalar decay constants $f_{\eta, \eta^{\prime}}^{8}$ and $f_{\eta, \eta^{\prime}}^{0}$ will be the same in both approaches since they are directly related to the physical measurements, while the inferred (theoretical) quantities $\tilde{f}_{8,0}$ and $f_{8,0}$ may differ in the fits due to the different approach taken.

As far as two-angle fits are concerned, we must stress here that the two mixing angles $\theta_{8}$ and $\theta_{0}$ introduced in Refs. [6, 15, 16] to parameterize the pseudoscalar decay constants are conceptually very different to the particle state mixing angle $\theta$ that rotates the flavour states $\left(\eta_{8}, \eta_{0}\right)$ into the physical states $(\eta, \eta \prime)$. However, because in our energy-dependent mixing angle scheme we do not distinguish between the mixing properties of the meson states from the mixing properties of the decay constants (see Sec. 2), a description of $\theta_{8}$ and $\theta_{0}$ in terms of $\theta_{\eta}$ and $\theta_{\eta^{\prime}}$, as done in Eq. (17), is allowed'.

\section{$4 \quad V$ - $P$ electromagnetic form factors in the energy- dependent mixing angle scheme}

In this section, we want to test some of the consequences of our approach. In particular, we are interested in the couplings of the radiative decays of lowestlying vector mesons, $V \rightarrow\left(\eta, \eta^{\prime}\right) \gamma$, and of the radiative decays $\eta^{\prime} \rightarrow V \gamma$, with

\footnotetext{
${ }^{7}$ The same statement in the usual energy-independent mixing angle scheme yields $\theta_{8}=$ $\theta_{0}=\theta$ and $\tilde{f}_{8,0}=f_{8,0}$.
} 
$V=\rho, \omega, \phi$. In order to predict such couplings we follow closely the method presented in Ref. [4] where the description of the light vector meson decays is based on their relation with the $A V V$ triangle anomaly, $A$ and $V$ being an axial-vector and a vector current respectively. The approach both includes $S U_{F}(3)$ breaking effects and fixes the vertex couplings $g_{V P \gamma}$ as explained below.

In that framework, one starts considering the correlation function

$$
i \int d^{4} x e^{i q_{1} x}\left\langle P\left(q_{1}+q_{2}\right)\left|T J_{\mu}^{\mathrm{EM}}(x) J_{\nu}^{V}(0)\right| 0\right\rangle=\epsilon_{\mu \nu \alpha \beta} q_{1}^{\alpha} q_{2}^{\beta} F_{V P \gamma}\left(q_{1}^{2}, q_{2}^{2}\right),
$$

where the currents are defined as

$$
\begin{gathered}
J_{\mu}^{\mathrm{EM}}=\frac{2}{3} \bar{u} \gamma_{\mu} u-\frac{1}{3} \bar{d} \gamma_{\mu} d-\frac{1}{3} \bar{s} \gamma_{\mu} s \\
J_{\mu}^{\rho, \omega}=\frac{1}{\sqrt{2}}\left(\bar{u} \gamma_{\mu} u \pm \bar{d} \gamma_{\mu} d\right) \quad \text { and } \quad J_{\mu}^{\phi}=-\bar{s} \gamma_{\mu} s
\end{gathered}
$$

The form factors values $F_{V P \gamma}(0,0)$ are fixed by the $A V V$ triangle anomaly (one $V$ being an electromagnetic current), and are written in terms of the pseudoscalar decay constants and the $\phi-\omega$ mixing angle $\theta_{V}$ as

$$
\begin{aligned}
& F_{\rho \eta \gamma}(0,0)=\frac{\sqrt{3}}{4 \pi^{2}} \frac{f_{\eta^{\prime}}^{0}-\sqrt{2} f_{\eta^{\prime}}^{8}}{f_{\eta^{\prime}}^{8} f_{\eta}^{8}-f_{\eta^{\prime}}^{8} f_{\eta}^{0}}, \\
& F_{\rho \eta \prime \gamma}(0,0)=\frac{\sqrt{3}}{4 \pi^{2}} \frac{f_{\eta}^{0}-\sqrt{2} f_{\eta}^{8}}{f_{\eta}^{0} f_{\eta^{\prime}}^{8}-f_{\eta}^{8} f_{\eta^{\prime}}^{0}}, \\
& F_{\omega \eta \gamma}(0,0)=\frac{1}{2 \sqrt{2} \pi^{2}} \frac{\left(c \theta_{V}-s \theta_{V} / \sqrt{2}\right) f_{\eta^{\prime}}^{0}-s \theta_{V} f_{\eta^{\prime}}^{8}}{f_{\eta^{\prime}}^{0} f_{\eta}^{8}-f_{\eta^{\prime}}^{8} f_{\eta}^{0}}, \\
& F_{\omega \eta \gamma}(0,0)=\frac{1}{2 \sqrt{2} \pi^{2}} \frac{\left(c \theta_{V}-s \theta_{V} / \sqrt{2}\right) f_{\eta}^{0}-s \theta_{V} f_{\eta}^{8}}{f_{\eta}^{0} f_{\eta^{\prime}}^{8}-f_{\eta}^{8} f_{\eta^{\prime}}^{0}}, \\
& F_{\phi \eta \gamma}(0,0)=-\frac{1}{2 \sqrt{2} \pi^{2}} \frac{\left(s \theta_{V}+c \theta_{V} / \sqrt{2}\right) f_{\eta^{\prime}}^{0}+c \theta_{V} f_{\eta^{\prime}}^{8}}{f_{\eta^{\prime}}^{0} f_{\eta}^{8}-f_{\eta^{\prime}}^{8} f_{\eta}^{0}}, \\
& F_{\phi \eta \prime \gamma}(0,0)=-\frac{1}{2 \sqrt{2} \pi^{2}} \frac{\left(s \theta_{V}+c \theta_{V} / \sqrt{2}\right) f_{\eta}^{0}+c \theta_{V} f_{\eta}^{8}}{f_{\eta}^{0} f_{\eta^{\prime}}^{8}-f_{\eta}^{8} f_{\eta^{\prime}}^{0}} .
\end{aligned}
$$

Using their analytic properties, we can express these form factors by a dispersion relation in the momentum of the vector current, which are then saturated with the lowest-lying resonances:

$$
F_{V P \gamma}(0,0)=\frac{f_{V}}{m_{V}} g_{V P \gamma}+\cdots,
$$

where the dots stand for higher resonances and multiparticle contributions to the correlation function. In the following we assume vector meson dominance (VMD) and thus neglect these contributions (see Ref. [4] for further details). 
The $f_{V}$ are the vector mesons' leptonic decay constants defined by

$$
\left\langle 0\left|J_{\mu}^{V}\right| V(p, \lambda)\right\rangle=m_{V} f_{V} \varepsilon_{\mu}^{(\lambda)}(p),
$$

where $m_{V}$ and $\lambda$ are the mass and the helicity state of the vector meson. The $f_{V}$ can be determined from the experimental decay rates [1] via

$$
\Gamma\left(V \rightarrow e^{+} e^{-}\right)=\frac{4 \pi}{3} \alpha^{2} \frac{f_{V}^{2}}{m_{V}} c_{V}^{2},
$$

with $c_{V}=\left(\frac{1}{\sqrt{2}}, \frac{s \theta_{V}}{\sqrt{6}}, \frac{c \theta_{V}}{\sqrt{6}}\right)$ for $V=\rho, \omega, \phi$. The experimental values are

$$
\begin{aligned}
f_{\rho^{0}} & =(216 \pm 5) \mathrm{MeV}, \\
f_{\omega} & =(180 \pm 3) \mathrm{MeV}, \\
f_{\phi} & =(244 \pm 4) \mathrm{MeV} .
\end{aligned}
$$

Finally, we introduce the vertex couplings $g_{V P \gamma}$, which are just the onshell $V$-P electromagnetic form factors:

$$
\left.\left\langle P\left(p_{P}\right)\left|J_{\mu}^{\mathrm{EM}}\right| V\left(p_{V}, \lambda\right)\right\rangle\right|_{\left(p_{V}-p_{P}\right)^{2}=0}=-g_{V P \gamma} \epsilon_{\mu \nu \alpha \beta} p_{P}^{\nu} p_{V}^{\alpha} \varepsilon_{V}^{\beta}(\lambda) .
$$

The decay widths of $P \rightarrow V \gamma$ and $V \rightarrow P \gamma$ are

$$
\begin{aligned}
& \Gamma(P \rightarrow V \gamma)=\frac{\alpha}{8} g_{V P \gamma}^{2}\left(\frac{m_{P}^{2}-m_{V}^{2}}{m_{P}}\right)^{3}, \\
& \Gamma(V \rightarrow P \gamma)=\frac{\alpha}{24} g_{V P \gamma}^{2}\left(\frac{m_{V}^{2}-m_{P}^{2}}{m_{V}}\right)^{3} .
\end{aligned}
$$

Eq. (22) allows us to identify the $g_{V P \gamma}$ couplings defined in (26) with the form factors $F_{V P \gamma}(0,0)$ listed in (21). We make use of this relationship to predict these couplings in the energy-dependent $\eta-\eta \prime$ mixing angle scheme. Our theoretical expectations are shown in Table [1. The couplings are expressed in terms of the mixing angle values $\theta_{\eta}$ and $\theta_{\eta^{\prime}}$, the octet and singlet pseudoscalar decay constants $f_{8}$ and $f_{0}$, the $\phi-\omega$ mixing angle $\theta_{V}$, and the corresponding vector decay constants $f_{V}$. We also include a numerical prediction for each coupling that should be compared with the experimental values extracted from (27) and [1]. In the numerical analysis we have included the deviation from ideal $\phi-\omega$ mixing by taking into account a value for the mixing angle of $\theta_{V}=38.6^{\circ}$ [1]. The error quoted in Table 1 does not reflect the full 


\begin{tabular}{|c|c|c|c|c|}
\hline$V$ & $P$ & $g_{V P \gamma}$ (th.) & & $g_{V P \gamma}($ exp.) \\
\hline$\rho$ & $\eta$ & $\frac{\sqrt{3} m_{\rho}}{4 \pi^{2} f_{\rho}} \frac{c \theta_{\eta^{\prime}} / f_{8}-\sqrt{2} s \theta_{\eta^{\prime}} / f_{0}}{c \theta_{\eta^{\prime}} c \theta_{\eta}+s \theta_{\eta^{\prime}} s \theta_{\eta}}$ & $=(1.43 \pm 0.10) \mathrm{GeV}^{-1}$ & $(1.47 \pm 0.28) \mathrm{GeV}^{-1}$ \\
\hline$\rho$ & $\eta \prime$ & $\frac{\sqrt{3} m_{\rho}}{4 \pi^{2} f_{\rho}} \frac{s \theta_{\eta} / f_{8}+\sqrt{2} c \theta_{\eta} / f_{0}}{c \theta_{\eta^{\prime}} c \theta_{\eta}+s \theta_{\eta^{\prime}} s \theta_{\eta}}$ & $=(1.23 \pm 0.11) \mathrm{GeV}^{-1}$ & $(1.31 \pm 0.06) \mathrm{GeV}^{-1}$ \\
\hline$\omega$ & $\eta$ & $\frac{m_{\omega}}{2 \sqrt{2} \pi^{2} f_{\omega}} \frac{\left(c \theta_{V}-s \theta_{V} / \sqrt{2}\right) c \theta_{\eta^{\prime}} / f_{8}-s \theta_{V} s \theta_{\eta^{\prime}} / f_{0}}{c \theta_{\eta^{\prime}} c \theta_{\eta}+s \theta_{\eta^{\prime}} s \theta_{\eta}}$ & $=(0.54 \pm 0.04) \mathrm{GeV}^{-1}$ & $(0.53 \pm 0.04) \mathrm{GeV}^{-1}$ \\
\hline$\omega$ & $\eta \prime$ & $\frac{m_{\omega}}{2 \sqrt{2} \pi^{2} f_{\omega}} \frac{\left(c \theta_{V}-s \theta_{V} / \sqrt{2}\right) s \theta_{\eta} / f_{8}+s \theta_{V} c \theta_{\eta} / f_{0}}{c \theta_{\eta^{\prime}} c \theta_{\eta}+s \theta_{\eta^{\prime}} s \theta_{\eta}}$ & $=(0.55 \pm 0.05) \mathrm{GeV}^{-1}$ & $(0.45 \pm 0.03) \mathrm{GeV}^{-1}$ \\
\hline$\phi$ & $\eta$ & $-\frac{m_{\phi}}{2 \sqrt{2} \pi^{2} f_{\phi}} \frac{\left(s \theta_{V}+c \theta_{V} / \sqrt{2}\right) c \theta_{\eta^{\prime}} / f_{8}+c \theta_{V} s \theta_{\eta^{\prime}} / f_{0}}{c \theta_{\eta^{\prime}} c \theta_{\eta}+s \theta_{\eta^{\prime}} s \theta_{\eta}}$ & $=(0.73 \pm 0.06) \mathrm{GeV}^{-1}$ & $(0.69 \pm 0.02) \mathrm{GeV}^{-1}$ \\
\hline$\phi$ & $\eta^{\prime}$ & $-\frac{m_{\phi}}{2 \sqrt{2} \pi^{2} f_{\phi}} \frac{\left(s \theta_{V}+c \theta_{V} / \sqrt{2}\right) s \theta_{\eta} / f_{8}-c \theta_{V} c \theta_{\eta} / f_{0}}{c \theta_{\eta^{\prime}} c \theta_{\eta}+s \theta_{\eta^{\prime}} s \theta_{\eta}}$ & $=(0.83 \pm 0.06) \mathrm{GeV}^{-1}$ & $(1.01 \pm 0.30) \mathrm{GeV}^{-1}$ \\
\hline
\end{tabular}

Table 1: Theoretical and experimental values of the on-shell $V$ - $\left(\eta, \eta^{\prime}\right)$ electromagnetic vertex couplings in the energy-dependent $\eta-\eta$ ' mixing angle scheme. For $g_{V P \gamma}$ (th.) we give the experimental errors coming from the decay constants $f_{P, V}$ and the mixing angle values $\theta_{\eta}$ and $\theta_{\eta \prime}$. We use $\theta_{V}=38.6^{\circ}$ for the $\phi-\omega$ mixing angle. Experimental values are taken from [[]].

theoretical uncertainty, but namely propagates the errors from (13) and (25). The agreement between our predictions and the experimental values is quite remarkable: all the values coincide at the $1 \sigma$ level except for the $g_{\omega \eta \prime \gamma}$ case. However, the $g_{\omega \eta / \gamma}$ coupling is rather sensitive to the $\phi-\omega$ mixing angle; for instance setting $\theta_{V}$ to the ideal mixing value of $35.3^{\circ}$ reduces the coupling by a $9 \%$. As seen from the table, the prediction for the decay $\phi \rightarrow \eta \gamma$ (which is largely independent of $\theta_{V}$ ) is in clear agreement with data, contrary to the encountered situation in the energy-independent mixing scheme where the $g_{\phi \eta \gamma}$ is only consistent with the experimental value for $\theta \simeq-22^{\circ}$ 四. The

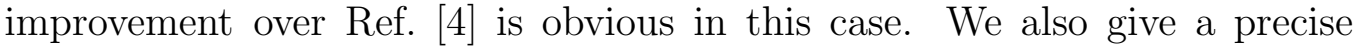
prediction for the $\phi \rightarrow \eta / \gamma$ decay, which is compatible with current data, although the experimental error is still too big to be conclusive.

Table 1 constitutes the main result of our work. Our analysis shows that the assumption of saturating the form factors $F_{V P \gamma}$ by lowest-lying resonances is satisfactory (a conclusion already reached in Ref. [4]), and that the energy-dependent $\eta-\eta \prime$ mixing angle scheme fits the data somewhat better than the energy-independent scheme does. To quantify this improvement, we have performed various fits to the full set of experimental data assuming, or not, the energy dependence of the $\eta-\eta \eta^{\prime}$ mixing angle. The results 


\begin{tabular}{|c|c|c|c|}
\hline Assumptions & Fitted data & Results & $\chi^{2} / d o f$ \\
\hline $\begin{array}{c}\theta_{\eta}=\theta_{\eta^{\prime}} \equiv \theta \\
f_{8}=1.28 f_{\pi}\end{array}$ & All data & $\begin{aligned} \theta & =(-18.1 \pm 1.2)^{\circ} \\
f_{0} & =(1.13 \pm 0.03) f_{\pi}\end{aligned}$ & $22.2 / 7$ \\
\hline $\begin{array}{c}\theta_{\eta}=\theta_{\eta^{\prime}} \equiv \theta \\
f_{8} \text { free }\end{array}$ & All data & $\begin{aligned} \theta & =(-18.1 \pm 1.2)^{\circ} \\
f_{8} & =(1.28 \pm 0.04) f_{\pi} \\
f_{0} & =(1.13 \pm 0.03) f_{\pi}\end{aligned}$ & $22.2 / 6$ \\
\hline $\begin{array}{c}\theta_{\eta} \neq \theta_{\eta^{\prime}} \\
f_{8}=1.28 f_{\pi}\end{array}$ & $\begin{array}{c}\Gamma(\eta, \eta \prime \rightarrow \gamma \gamma)+ \\
R_{J / \psi}\end{array}$ & $\begin{aligned} \theta_{\eta} & =(-6.5 \pm 2.5)^{\circ} \\
\theta_{\eta^{\prime}} & =(-23.1 \pm 3.0)^{\circ} \\
f_{0} & =(1.31 \pm 0.07) f_{\pi}\end{aligned}$ & not applicable \\
\hline $\begin{array}{c}\theta_{\eta} \neq \theta_{\eta^{\prime}} \\
f_{8}=1.28 f_{\pi}\end{array}$ & All data & $\begin{array}{c}\theta_{\eta}=(-6.9 \pm 2.1)^{\circ} \\
\theta_{\eta^{\prime}}=(-24.6 \pm 2.3)^{\circ} \\
f_{0}=(1.34 \pm 0.07) f_{\pi}\end{array}$ & $8.4 / 6$ \\
\hline $\begin{array}{c}\theta_{\eta} \neq \theta_{\eta^{\prime}} \\
f_{8} \text { free }\end{array}$ & All data & $\begin{aligned} \theta_{\eta} & =(-5.7 \pm 2.7)^{\circ} \\
\theta_{\eta^{\prime}} & =(-24.6 \pm 2.3)^{\circ} \\
f_{8} & =(1.32 \pm 0.06) f_{\pi} \\
f_{0} & =(1.37 \pm 0.07) f_{\pi}\end{aligned}$ & $7.9 / 5$ \\
\hline
\end{tabular}

Table 2: Results for the $\eta-\eta$ mixing angle and decay constants in the energyindependent and -dependent mixing angle schemes. For every fit, the theoretical assumptions taken, the set of fitted experimental data, and the value of the $\chi^{2} /$ dof are shown in the first, second and last column respectively. Numerical results are presented in the third column. All data stands for the decay widths of $\left(\eta, \eta^{\prime}\right) \rightarrow \gamma \gamma, V \rightarrow P \gamma, P \rightarrow V \gamma$, and the ratio $R_{J / \psi}$. 
are presented in Table 2. To check the consistency of our approach we have extended the fit in Eq. (13) (third data row in Table 2) to include all experimental data that account not only for the decay widths $\left(\eta, \eta^{\prime}\right) \rightarrow \gamma \gamma$ and the ratio $R_{J / \psi}$ but also the radiative decay widths of $V \rightarrow P \gamma$ and $P \rightarrow V \gamma$. The theoretical constraint $f_{8}=1.28 f_{\pi}$ is also relaxed in order to test the stability of the result. As seen from Table 2, a significant enhancement in the $\chi^{2} / d o f$ is achieved when the constrain $\theta_{\eta}=\theta_{\eta}$ is relaxed, allowing us to show explicitly the improvement of our analysis with respect to the one in Ref. 俰.

\section{Conclusions}

In this letter, we have performed a phenomenological analysis on various decay processes assuming an energy dependence of the $\eta-\eta$ ' mixing angle as the main work hypothesis. We have been mainly interested in the consequences of such hypothesis on the electromagnetic couplings of $V \rightarrow P \gamma$ and $P \rightarrow V \gamma$ processes. The radiative decays $\left(\eta, \eta^{\prime}\right) \rightarrow \gamma \gamma$ together with the ratio $R_{J / \psi}$ have been used to fit the values of the mixing angle and pseudoscalar decay constants. Using the description of vector meson decays in terms of their relation with the $A V V$ triangle anomaly, a theoretical prediction for the $g_{V P \gamma}$ couplings have been derived. The agreement between our theoretical predictions and the experimental values is quite remarkable and can be considered as a consistency check of the whole approach. We have also established the link with the two-angle approaches of Refs. [6, 15, 16].

\section{Acknowledgments}

This work was supported by the IISN (Belgium) and by the Communauté Française de Belgique (Direction de la Recherche Scientifique programme $\mathrm{ARC})$.

\section{References}

[1] Review of Particle Properties, Particle Data Group, C. Caso et al., Eur. Phys. J. C3, 1 (1998). 
[2] F. J. Gilman and R. Kauffman, Phys. Rev. D36, 2761 (1987).

[3] A. Bramon and M. D. Scadron, Phys. Lett. B234, 346 (1990).

[4] P. Ball, J.-M. Frère and M. Tytgat, Phys. Lett. B365, 367 (1996).

[5] A. Bramon, R. Escribano and M. D. Scadron, Phys. Lett. B403, 339 (1997), and Eur. Phys. J. C7, 271 (1999).

[6] H. Leutwyler, Nucl. Phys. Proc. Suppl. 64, 223 (1998).

[7] P. Herrera-Siklódy et al., Nucl. Phys. B497, 345 (1997).

[8] P. Herrera-Siklódy et al., Phys. Lett. B419, 326 (1998).

[9] K. Maltman, Phys. Lett. B313, 203 (1993).

[10] R. Akhoury and J.-M. Frère, Phys. Lett. B220, 258 (1989).

[11] J. F. Donoghue, E. Golowich and B. R. Holstein, Dynamics of the Standard Model, Cambridge University Press (1992).

[12] A. V. Kisselev and V. A. Petrov, Z. Phys. C58, 595 (1993).

[13] P. Castoldi and J.-M. Frère, Z. Phys. C40, 283 (1988).

[14] V. A. Novikov, M. A. Shifman, A. I. Vainshtein and V. I. Zakharov, Nucl. Phys. B165, 55 (1980).

[15] R. Kaiser and H. Leutwyler, hep-ph/9806336.

[16] Th. Feldmann and P. Kroll, Eur. Phys. J. C5, 327 (1998). 